\title{
ATLAS Status and First Results
}

\author{
P. Conde Muíño on behalf of the ATLAS Collaboration
}

LIP, Av. Elias Garcia, 14, 1000-149 Lisboa, Portugal

\begin{abstract}
.
After more than 20 years of continuous work and several months of commissioning with cosmic muon data, the ATLAS experiment started data taking at the Large Hadron Collider (CERN). From November 2009 to May 2010, ATLAS registered proton-proton collisions at $900 \mathrm{GeV}, 2.36 \mathrm{GeV}$ and finally $7 \mathrm{TeV}$ center of mass energy. These data samples have been used to study the detector performance in detail and are currently being used also for the first physics studies. In this document, we present some selected physics and performance results obtained with the first $7 \mathrm{TeV}$ protonproton collisions.
\end{abstract}

Keywords: ATLAS, LHC, detector performance, first physics results, $7 \mathrm{TeV}$ collisions

PACS: 10,29

\section{INTRODUCTION}

ATLAS is one of the four LHC experiments, designed to study a wide range of physics processes that go from detailed studies of the electroweak symmetry breaking mechanism (i.e. the search for the Higgs boson), precision measurements of the Standard Model (SM) processes and searches for new physics mechanisms.

After many years of design, construction and commissioning work, ATLAS has seen the first proton-proton collisions delivered by the LHC in November 2009, at a center of mass energy of $900 \mathrm{GeV}$. Later, the LHC energy increased up to $2.36 \mathrm{TeV}$ first and up to $7 \mathrm{TeV}$ afterwards. Since then and for a period of about 2 months, ATLAS collected $14.85 \mathrm{nb}^{-1}$ of integrated luminosity. In this period, the record instantaneous luminosity was $2.4 \cdot 10^{29} \mathrm{~Hz} / \mathrm{cm}^{2}$, that is equivalent to about $8 \mathrm{~W} \rightarrow \mu \nu$ events per hour.

Our main goals with this first $7 \mathrm{TeV}$ data are the commissioning, calibration and alignment of the detector with well known physics samples, on one hand, and the first SM physics studies on the other. With the collected integrated luminosity, and taking into account the LHC energy, we are at the level of doing b-physics studies, seeing high energetic jets or $\mathrm{W}$ bosons decaying into the leptonic channels, in addition to minimum bias studies. For the commissioning of the detector, $J / \Psi$ and $\Upsilon$ decays to leptons, photon conversions, $K_{S}^{0}$ and $\Lambda^{0}$ decays, ... can be used to study the momentum/energy scale and resolutions, material distributions in front of the calorimeters, lepton trigger and reconstruction efficiency, ...

In this paper we will present some selected results from these first physics and performance studies. The paper starts with a description of the ATLAS detector, followed by b-tagging performance studies, prompt electron and muon studies, $J / \Psi$ properties and observation of $W, Z$ bosons.

CP1317, QCD@WORK 2010, International Workshop on Quantum Chromodynamics: Theory and Experiment edited by L. Angelini, G. E. Bruno, P. Colangelo, D. Creanza, F. De Fazio, and E. Nappi (C) 2010 American Institute of Physics 978-0-7354-0872-2/10/\$30.00 


\section{THE ATLAS SPECTROMETER}

ATLAS is a toroidal apparatus with a typical onion-like structure. Going from the inner to the outermost layers it is made of three tracking detectors (called all together Inner Detector or ID) immerse in a solenoidal 2T magnetic field, a calorimeter system composed of electromagnetic and hadronic calorimeters and the muon chambers immerse in a magnetic field provided by 8 air-core toroid magnets. The ATLAS coordinate system is defined as follows: the origin is the nominal interaction point, the positive $\mathrm{x}$-axis points to the center of the LHC ring, the y-axis points upwards and the $\mathrm{z}$-axis is parallel to the LHC ring. The pseudo-rapidity, $\eta$, is defined as $\eta=-\ln \tan (\theta / 2)$, where $\theta$ is the polar angle. A detailed description of the ATLAS detector can be found in [1], [2].

The ATLAS Inner Detector has been designed to provide high precision tracking, momentum and vertex measurements, with high granularity at the inner radii followed by continuous tracking at the outer radii. The high granularity is obtained with three layers of pixel detectors, followed by four double layers of silicon strip detectors (SCT). The continuous tracking is made of straw tubes (TRT) that produce an average of 36 hits per track. The straw tubes are filled with transition radiation gas providing additional electron/pion separation in the momentum range between $0.5-100 \mathrm{GeV}$. The resolution in momentum is $\sigma\left(p_{T}\right) / p_{T}=0.05 \% \times p_{T} / \mathrm{GeV} \oplus 1 \%$ and in impact parameter $\sigma\left(d_{0}\right)=$ $10 \mu m \oplus 140 \mu m /\left(p_{T} / G e V\right)$.

The ATLAS electromagnetic calorimeter is made of liquid Argon (LAr) as ionization material and lead as absorber, with accordion geometry. It has high granularity and longitudinal segmentation, with nearly $200 \mathrm{~K}$ channels, in order to identify electrons/photons and provide good position and energy resolution $(10 \% / \sqrt{E / G e V}+$ $0.7 \%)$. The hadronic calorimeter is made of scintillating tiles and iron as absorber in the region up to $|\eta|<3.2$, providing an energy resolution of $\sigma / E \sim 50 \% / \sqrt{E / G e V} \oplus 0.03$ for single pions, and uses the LAr technology in the forward region, with copper as absorber. The good hermiticity of the calorimeters (covers up to $|\eta| \sim 5$ ) ensures good missing transverse energy measurement.

The muon system in ATLAS uses different technologies for the trigger and offline reconstruction. The trigger system is composed of 6 layers of Resistive Plate Chambers (RPC) in the central region $(|\eta|<1)$ and seven layers of Thin Gap Chambers (TGC) in the region $1<|\eta|<2.4$. For precise offline spacial muon measurement we have Monitored Drift Tubes in the central region $(|\eta|<2.7)$. The region between $2<|\eta|<$ 2.7 is equipped additionally with four layers of Cathode Strip Chambers (CSC) in order to cope with the demanding conditions of the LHC.

\section{EARLY PHYSICS STUDIES}

\section{Reconstruction of resonances}

We have reconstructed several different mass resonances $\left(K_{S}^{0}, \Lambda^{0}, D^{0}, D^{*}, \Omega, \ldots\right)[8]$ [10]. The study of these resonances is interesting not only for the modeling of the underlying event and as tests of QCD predictions but also because they provide useful 
TABLE 1. $K_{S}^{0}$ and $\Lambda^{0}$ reconstructed mass and width (i.e. width at half maximum of the signal peak divided by 2.35).

\begin{tabular}{|c|c|c|c|c|c|}
\hline & \multicolumn{2}{|c}{ Barrel } & \multicolumn{2}{c|}{ Endcap } & PDG \\
\hline & mean [MeV] & width $[\mathrm{MeV}]$ & mean $[\mathrm{MeV}]$ & width $[\mathrm{MeV}]$ & mass [MeV] \\
\hline$K_{S}^{0}$ Data & $497.427 \pm 0.006$ & 5.60 & $497.797 \pm 0.016$ & 10.45 & $497.614 \pm 0.024$ \\
\hline$K_{S}^{0} \mathrm{MC}$ & $497.329 \pm 0.006$ & 5.42 & $497.868 \pm 0.016$ & 10.14 & $497.614 \pm 0.024$ \\
\hline$\Lambda^{0}$ Data & $1115.73 \pm 0.01$ & 2.28 & $1115.78 \pm 0.02$ & 3.84 & $1115.683 \pm 0.006$ \\
\hline$\Lambda^{0} \mathrm{MC}$ & $1115.768 \pm 0.01$ & 2.18 & $1115.78 \pm 0.02$ & 3.68 & $1115.683 \pm 0.006$ \\
\hline$\overline{\Lambda^{0} \text { Data }}$ & $1115.79 \pm 0.01$ & 2.32 & $1115.79 \pm 0.02$ & 3.87 & $1115.683 \pm 0.006$ \\
\hline$\overline{\Lambda^{0} \mathrm{MC}}$ & $1115.71 \pm 0.01$ & 2.19 & $1115.79 \pm 0.02$ & 3.69 & $1115.683 \pm 0.006$ \\
\hline
\end{tabular}

tools to understand the detector performance. The mass distributions of the $K_{S}^{0}, \Lambda^{0}$ resonances were fit separately for the barrel and extended barrel. A table with the resulting mean positions and widths is shown in table 1, comparing the results in data and MC, and comparing to the PDG values[11]. There is a good agreement in the mean mass and resolutions between MC simulation and real data, for different regions of the ATLAS detector, meaning that the magnetic field modeling and momentum scale are well simulated.

\section{b-tagging performance}

Identifying jets produced by b-quarks is an essential tool for many physics studies, like precision measurements of the top quark properties, searches for the Higgs boson in the low mass regime or physics beyond the Standard Model since it helps reducing the background from light jets.

The performance of the ATLAS b-tagging algorithms is being studied in detail [6],[7]. One of the methods being used, exploits the large impact parameter that characterize the tracks coming from heavy flavor particle decays. A signed impact parameter is defined in such a way that tracks coming from the primary interaction point have equal probability of positive/negative signs, while tracks coming from secondary vertices mainly have large positive impact parameters. The distribution of the signed impact parameter significance is shown in figure 1 (left) for real data and MC. The overall agreement data-MC is good, although the $\mathrm{MC}$ has a slightly narrower distribution, due to residual misalignments in the data. For the MC, we have separated the different contributions: light jets, that produce a distribution that is rather symmetric, and $\mathrm{c}$ and b-jets, that have large tails to the positive side, as expected. The tails in the positive side of the light jet distribution is due to kaon/pion decays and $V^{0}$ decays that were not removed.

The negative side of the impact parameter significance distribution from data can be used to define the probability density function that a track comes from the primary vertex. Combining the probabilities for all the tracks in a jet we can define the probability that a jet is compatible with a light jet, as shown in figure 1 (right). The jet probability 

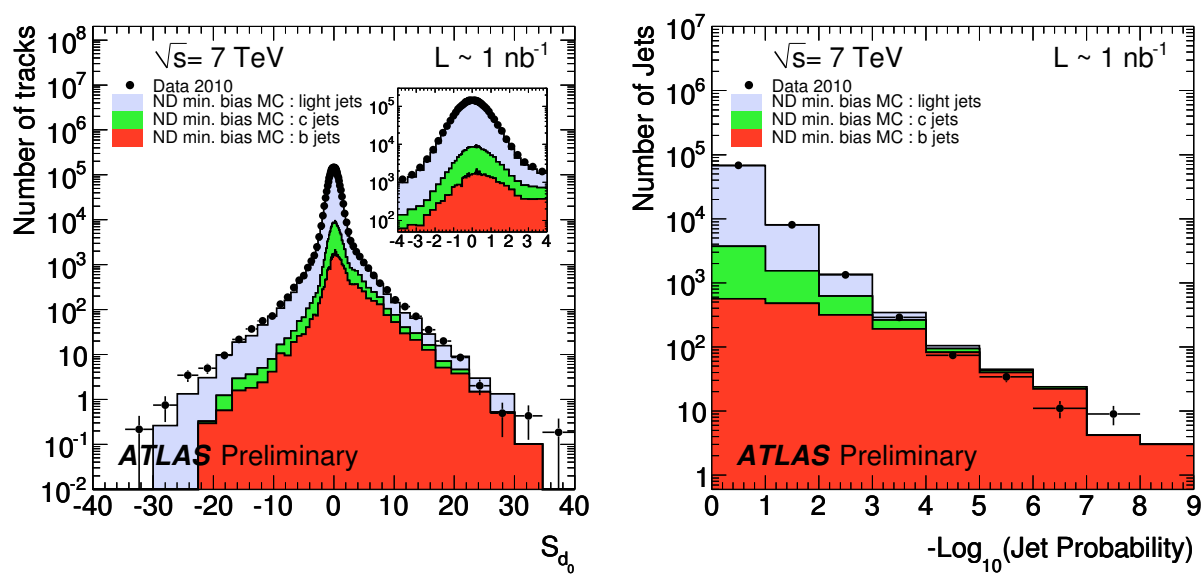

FIGURE 1. Left: signed impact parameter significance distribution. Right: distribution of the negative logarithm of the probability that a jet is compatible with a light jet. In both cases, the data is shown with black points while the MC is the full histogram.

distribution is consistent with the one obtained from the Minimum Bias MC. The plot shows that for very small probabilities the sample is dominated by $\mathrm{c}$ and $\mathrm{b}$ jets, while the light jets mainly have larger probabilities. We therefore expect that applying selection cuts based on this jet probability will lead to similar efficiencies in data and in MC. These results are encouraging, since they mean that we will soon be able to use b-tagging for physics studies.

\section{Prompt electron studies}

A precise understanding of the inclusive electron production at the LHC is very important since it is the main background for processes involving high $E_{T}$ electrons in the final state. Prompt electrons have to be separated from QCD hadrons and from electrons originating from photon conversions. The identification of the electron candidates can be done using the shower shape variables, the leakage in the hadronic calorimeter, quality cuts in the track reconstruction and in the matching between the calorimeter cluster and the track [12]. These are called medium identification criteria. Then, to separate prompt electrons from QCD hadrons and secondary electrons, two variables can be used: the fraction of high threshold hits in the TRT detector (produced by the transition radiation when an electron crosses the straw tubes) and the number of hits in the silicon detectors. The distribution of these two variables can be seen in figure 2 for data and MC. There is a good agreement between data and MC in the shape of these two distributions. The contributions from the three different sources are differentiated in the MC plots, showing that the fraction of high threshold hits can be used to separate hadrons and the number of hits in the first pixel layer is a very good variable to separate the secondary electrons. 

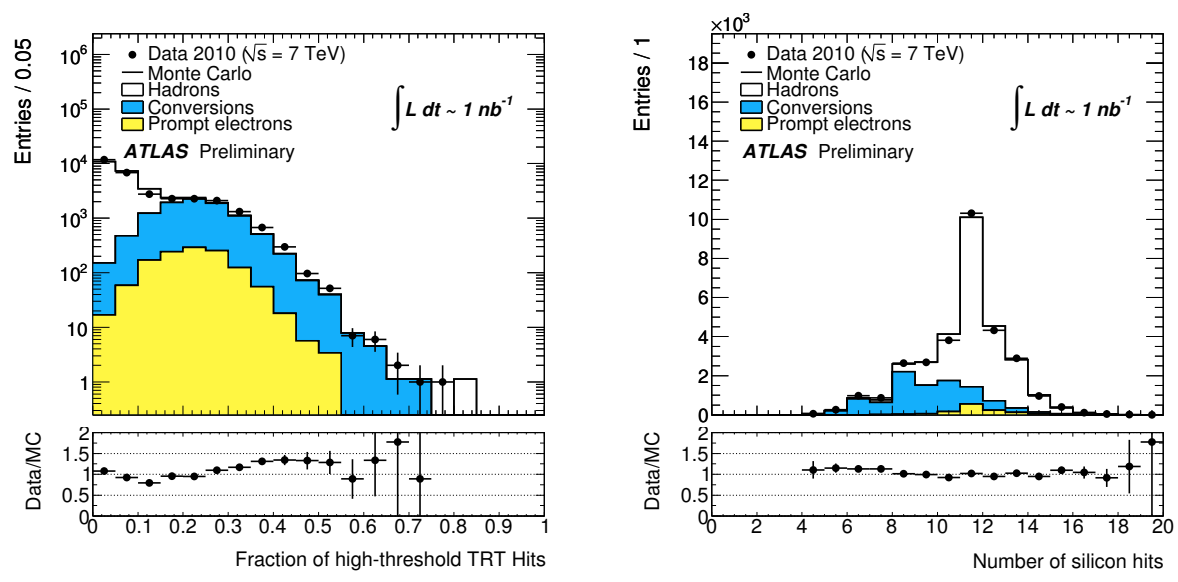

FIGURE 2. Distribution of the fraction of high threshold TRT hits (left) and number of hits in the silicon detectors (right) for the data (points) and the MC (full histogram). The different MC components are identified: hadrons, prompt electrons and conversion.
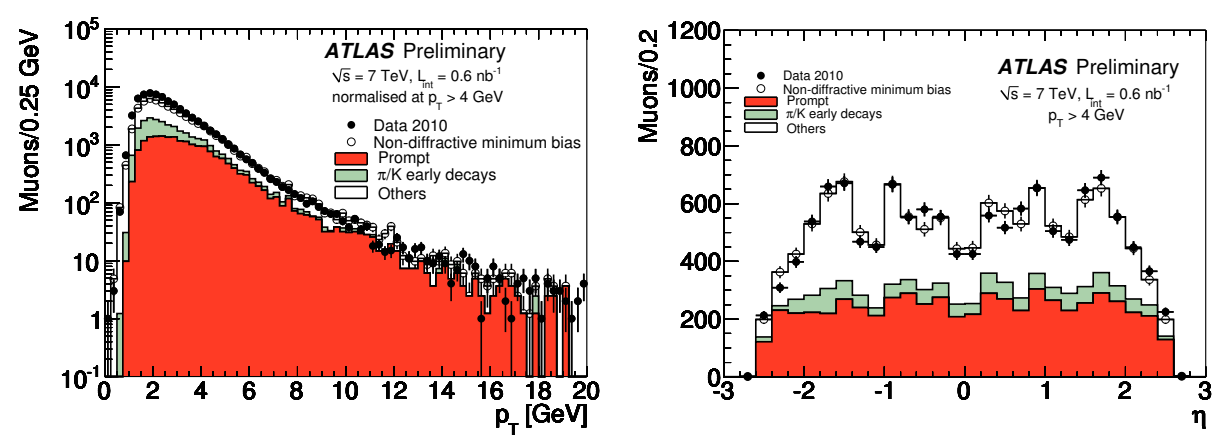

FIGURE 3. Left: muon $p_{T}$ spectrum. Right: pseudo-rapidity distribution for muon candidates with $p_{T}>4 \mathrm{GeV}$.In both cases data is shown with points while MC is shown with the full histogram (dark color for prompt muons, light color for $\pi / \mathrm{K}$ early decays and white for $\pi / \mathrm{K}$ late decays and fakes.

\section{Prompt muon studies}

Muon tracks in ATLAS can be reconstructed using different algorithms. In the study presented here [5] the muons were reconstructed as a combined track in the ID and Muon Spectrometer. The $p_{T}$ spectrum of the muon candidates is shown in figure 3 (left). For the $\mathrm{MC}$, the different contributions are separated: prompt muons (that include muons from Drell-Yan, $\mathrm{b}$ and $\mathrm{c}$ decays), early $\pi / \mathrm{K}$ decays and others (including mainly late $\pi / \mathrm{K}$ decays and fakes). For the region with $p_{T}<4 \mathrm{GeV}$ the spectrum is dominated by background from $\pi / \mathrm{K}$ decays. In the region above $4 \mathrm{GeV}$ the signal contribution is about $50 \%$ and the MC spectrum is consistent with the real data. 

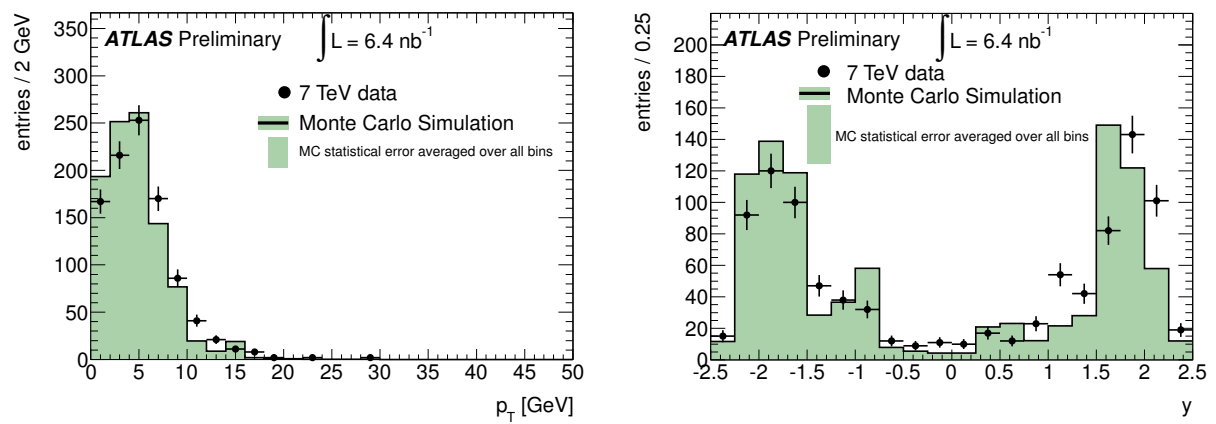

FIGURE 4. $p_{T}$ (left) and rapidity (right) distributions of the $J / \Psi \rightarrow \mu \mu$ candidates. Data is shown with full points and $\mathrm{MC}$ with the shadowed histogram.

The pseudo-rapidity distribution for muons with $p_{T}>4 \mathrm{GeV}$ is shown in figure 3 (right). The contribution from early decays and prompt muons are flat in $\eta$. The late decays show a more complex structure since $\pi$ and K decays in the calorimeter are much more sensitive to the material inhomogeneity. The good agreement between data and MC means good modeling of the detector acceptance and reconstruction efficiency.

\section{$J / \Psi$ reconstruction}

$J / \Psi$ and $\Upsilon$ production provide sensitive tests of QCD predictions. In addition they are essential for B-physics studies and to understand the detector performance (trigger, tracking and momentum/energy scale) in the low $p_{T}$ regime. The $J / \Psi$ peak has been reconstructed in the $\mu \mu$ decay channel [13]. The mean value of the $J / \Psi$ mass, as obtained with an unbinned maximum likelihood fit, is $3.095 \pm 0.004 \mathrm{GeV}$, in good agreement with PDG and with the MC simulation. The $p_{T}$ and rapidity distributions of the $J / \Psi \rightarrow \mu \mu$ candidates is shown in figure 4 . The $J / \Psi$ candidates have mainly very small $p_{T}$ (few $\mathrm{GeV}$ ). With that low $p_{T}$, it is more likely that they are reconstructed in the forward region, where the total momentum is larger, as one can see in figure 4 (right).

\section{Observation of $\mathrm{W}$ and $\mathrm{Z}$ bosons}

$\mathrm{W}$ and $\mathrm{Z}$ bosons are expected to be produced copiously at the LHC and for the first time at an unexplored energy regime. These events will be used to improve the precision measurements of the $\mathrm{W}$ boson properties, to perform stringent tests of non-perturbative QCD effects and to constraint the Parton Density Functions at the LHC energies.

A preselection of events with hight $p_{T}$ leptons $(\mathrm{e}, \mu)$ was done by requiring a combined muon with $p_{T}>15 \mathrm{GeV}$ or an electromagnetic cluster loosely identified (i.e. using only shower shape variables for the identification) with $E_{T}>20 \mathrm{GeV}$ [14]. Event cleaning requirements to reject badly reconstructed jets and improve the missing trans- 

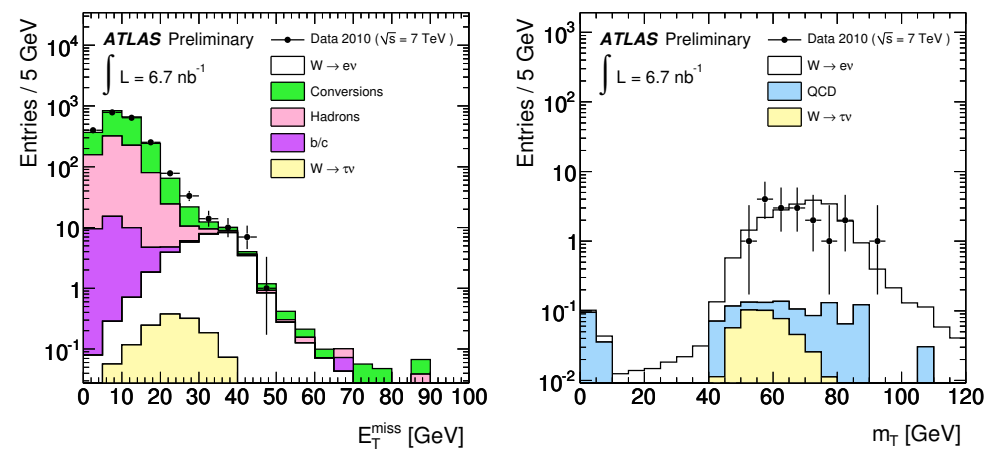

FIGURE 5. Left: missing transverse energy distribution for all the events that pass the preselection. Right: transverse mass distribution for the $W \rightarrow e v$ candidates after the full selection. . Data is shown with points and the different MC contributions with the full histograms.

verse energy $\left(E_{\mathrm{T}}^{\text {miss }}\right)$ measurements were also applied [15]. The $E_{\mathrm{T}}^{\text {miss }}$ distribution for the selected events in the electron channel is shown in figure 5 (left), for data and MC, where the MC was normalized to the real data. There is an overall good agreement between data and $\mathrm{MC}$ in the shape of the distributions. At about $40 \mathrm{GeV}$ a visible excess of events coming from the $W \rightarrow e v$ decays is clearly visible.

The $W$ candidate selection is completed with tight electron identification, muon/electron isolation conditions, large missing transverse energy and lepton $p_{T}$ requirements $\left(E_{\mathrm{T}}^{\text {miss }}>25 \mathrm{GeV}, p_{T}^{l}>20 \mathrm{GeV}\right)$. The candidates with transverse mass larger than $40 \mathrm{GeV}$ surviving the selection are 17 events for the $W \rightarrow e v$ channel and 40 for the $W \rightarrow \mu v$ channel. The transverse mass distribution for the $e v$ channel can be seen in figure 5 (right). The dominant backgrounds are expected to be QCD for the electron channel and $Z \rightarrow \mu \mu$ for the muon channel.

The expected number of events for these selection conditions is $23.1 \pm$ 1.2 (stat) \pm 1.7 (syst) \pm 4.6 (lumi) and $28.7 \pm 0.5$ (stat) \pm 3.9 (syst) \pm 5.7 (lumi) in the electron and muon channels respectively. The expected number of background events is $2.4 \pm 1.2$ (stat) \pm 0.4 (syst) \pm 0.5 (lumi) in the $e v$ channel and $2.8 \pm$ 0.5 (stat) \pm 0.8 (syst) \pm 0.6 (lumi) in the $\mu v$ channel, where data driven methods were used to estimate the QCD contribution and the MC simulation was used for the other backgrounds.

$\mathrm{Z}$ bosons were searched for in a sample of $6.7 \mathrm{nb}^{-1}$ of integrated luminosity for the $e^{+} e^{-}$channel and $7.9 \mathrm{nb}^{-1}$ for the $\mu^{+} \mu^{-}$channel. The preselection was done in the same way as for the $\mathrm{W}$ observation studies. The lepton identification, however, was done using softer conditions than in the previous analysis: in the muon channel, one of muons had to pass the same conditions as for the $\mathrm{W}$ analysis, but for the other one the $p_{T}$ requirement was softer $\left(p_{T}>15 \mathrm{GeV}\right)$. In addition, the same isolation condition was used for both muons. On the electron channel the medium identification criteria was used, and both electrons were required to have $E_{T}>20 \mathrm{GeV}$. No $E_{\mathrm{T}}^{\text {miss }}$ condition was imposed. The number of $\mathrm{Z}$ candidates that passed these requirements, in the invariant 
mass window $m_{l l}=80-100 \mathrm{GeV}$ were one in the electron channel and 2 in the muon channel. The expected number of signal events is $1.6 \pm 0.1$ (syst) \pm 0.3 (lumi) for the $e^{+} e^{-}$channel and 3.2 \pm 0.8 (syst) \pm 0.6 (lumi) for the $\mu^{+} \mu^{-}$channel, where the largest uncertainty comes from the lepton reconstruction efficiencies. The expected number of background events in this mass window is 0.01 for the electron channel and below that for the muon channel.

\section{SUMMARY AND CONCLUSIONS}

After a spectacular re-start of the LHC, ATLAS has recorded about $15 \mathrm{nb}^{-1}$ of integrated luminosity at $7 \mathrm{TeV}$ center of mass energy, in about 7 weeks. This data is being intensively used for detector performance and physics studies. The ATLAS detector is performing remarkably well. We have a good understanding of the momentum scale, material and track parameters. We also have good understanding of the electron, photon, muon, jet and missing transverse energy reconstruction and the overall description of the detector in the MC simulation is good. ATLAS is starting now to rediscover the Standard Model, and many physics results are currently being prepared.

\section{ACKNOWLEDGMENTS}

The author wants to acknowledge the support from FCT (Portugal) co-financed by QREN/COMPETE of ERDF fund (European Union).

\section{REFERENCES}

1. The ATLAS Collaboration, JINST 3 S08003, 2008.

2. ATLAS Collaboration, G Aad et al, CERN-OPEN-2008-020 (2008).

3. C. Amsler et al. (particle Data Group), Physics letters B667, 1 (2008), Section 27.3.

4. D. Grandjean, G. Gaycken, ECFA Workshop Durham, September 2004.

5. The ATLAS Collaboration, ATLAS public note ATLAS-CONF-2010-035, June 2010.

6. The ATLAS Collaboration, ATLAS public note ATLAS-CONF-2010-041, June 2010.

7. The ATLAS Collaboration, ATLAS public note ATLAS-CONF-2010-042, June 2010.

8. The ATLAS Collaboration, ATLAS public note ATLAS-CONF-2010-033, June 2010.

9. The ATLAS Collaboration, ATLAS public note ATLAS-CONF-2010-032, June 2010.

10. The ATLAS Collaboration, ATLAS public note ATLAS-CONF-2010-034, June 2010.

11. C. Amsler et al. (Particle Data Group), Physics Letters B667, 1 (2008) and 2009 partial update for the 2010 edition.

12. ATLAS public page https://twiki.cern.ch/twiki/bin/view/Atlas/AtlasResults.

13. The ATLAS Collaboration, ATLAS public note ATLAS-CONF-2010-045, June 2010.

14. The ATLAS Collaboration, ATLAS public note ATLAS-CONF-2010-044, June 2010.

15. The ATLAS Collaboration, ATLAS public note ATLAS-CONF-2010-038, June 2010. 